\title{
INFINITE SYSTEMS OF INTEGRAL EQUATIONS IN FRÉCHET SPACES USING THE NOTION OF L-FUNCTIONS
}

\author{
MOHSEN HOSSEINZADEH MOGHADDAM, REZA ALLAHYARI, \\ MOHSEN ERFANIAN OMIDVAR, AND MAHMOUD HASSANI
}

Received 09 March, 2017

\begin{abstract}
The aim of this paper is to prove some fixed point theorems for L-functions with the help of measure of noncompactness and the Tychonoff fixed point theorem. Also, we prove some existence theorems for a general infinite system of integral equations. As an application, we study the problem of the existence of solutions for infinite systems of nonlinear integral equations of Hammerstein type in two variables. The results obtained extend several ones. Finally, an example is presented to guarantee our results.
\end{abstract}

2010 Mathematics Subject Classification: 47H09; 47H10; 54H25

Keywords: measure of noncompactness, Fréchet spaces, Tychonoff fixed point theorem, infinite systems of equations

\section{INTRODUCTION AND PRELIMINARIES}

There are three major branches of fixed point theory in functional analysis, and each branch has its celebrated theorems. In the topological branch of fixed point theory, the main three theorems are Brouwer's fixed point theorem, its infinite dimensional version, Schauder's fixed point theorem, and Tychonoff fixed point theorem on locally convex spaces which in each of them compactness plays an essential role.

Theorem 1 (Schauder [1]). Let $C$ be a closed and convex subset of a Banach space $E$. Then every compact and continuous map $F: C \longrightarrow C$ has at least one fixed point.

Theorem 2 (Tychonoff fixed point theorem [1]). Let E be a Hausdorff locally convex linear topological space, $C$ a convex subset of $E$ and $F: C \longrightarrow E$ a continuous mapping such that

$$
F(C) \subseteq A \subseteq C
$$

with A compact. Then $F$ has at least one fixed point.

The concept of measure of noncompactness together with the well-known Darbo's fixed point theorem have played a basic role in nonlinear functional analysis, especially in topological fixed point theory. Up to now, several papers have been published 
on the existence and behavior of solutions of nonlinear differential and integral equations, using the technique of measure of noncompactness together with the Darbo's fixed point theorem. Some of these works are noted in [3, 4, 7, 8, 10, 12-16, 18, 19]. Recently, the theory of infinite systems of integral or differential equations can also be used in solving some problems for differential and integral equations (see [5,11, 22-25]).

On the other hand, In 1969, Meir and Keeler [21] proved the following very interesting fixed-point theorem, which is a generalization of the Banach contraction principle [6].

Definition 1 ([21]). Let $(X, d)$ be a metric space. Then a mapping $\mathrm{T}$ on $\mathrm{X}$ is said to be a Meir-Keeler contraction (MKC, for short) if for any $\varepsilon>0$, there exists $\delta>0$ such that

for all $x, y \in X$.

$$
\varepsilon \leq d(x, y)<\varepsilon+\delta \Longrightarrow d(T x, T y)<\varepsilon,
$$

Theorem 3 (Meir and Keeler [21]). Let $(X, d)$ be a complete metric space. If $T: X \longrightarrow X$ is a Meir-Keeler contraction, then T has a unique fixed point.

Next, Lim [20] and Suzuki [26] introduced the notion of L-functions and characterized Meir-Keeler contractions in metric spaces. Moreover, Aghajani et al. [2] introduce the notion of Meir-Keleer condensing operator on a Banach space, a characterization using strictly L-functions and provide a few generalizations of Darbo's fixed point theorem. In this paper, we state and prove some fixed point theorems for L-functions on Fréchet spaces with the help of measure of noncompactness and the Tychonoff fixed point theorem, which is an extension of the results [21], Lim [20], Suzuki [26] and Darbo [13]. Then, we prove some existence theorems for a general infinite system of integral equations. Finally, using the obtained results, we are going to study the existence of continuous solutions of the infinite system of nonlinear integral equations of Hammerstein type

$$
=f_{n}\left(t, s, x_{1}(t, s) \ldots, x_{n}(t, s), \int_{0}^{\infty} \int_{0}^{\infty} k_{n}(t, s, v, w) h_{n}\left(v, w,\left(x_{j}(v, w)\right)_{j=1}^{\infty}\right) d v d w\right),
$$

$t, s \in \mathbb{R}_{+}, n \in \mathbb{N}$. The functions $f_{n}, k_{n}$ and $h_{n}(n \in \mathbb{N})$ are continuous and satisfy some certain conditions, specified later. Furthermore, an example is presented to guarantee our results.

Now, we recall some basic facts concerning measures of noncompactness. Denote by $\mathbb{R}$ the set of real numbers and put $\mathbb{R}_{+}=[0,+\infty)$. Let $(E,\|\cdot\|)$ be a real Banach space with zero element 0 . Let $\bar{B}(x, r)$ denote the closed ball centered at $x$ with radius $r$. The symbol $\bar{B}_{r}$ stands for the ball $\bar{B}(0, r)$. For $X$, a nonempty subset of $E$, we denote by $\bar{X}$ and $\operatorname{Conv} X$ the closure and the closed convex hull of $X$, respectively. Moreover, let us denote by $\mathfrak{M}_{E}$ the family of nonempty bounded subsets of $E$ and by $\mathfrak{N}_{E}$ its subfamily consisting of all relatively compact sets. 
A topological vector space (TVS) is a vector space $X$ over the field $\mathbb{R}$ which is endowed with a topology such that the maps $(x, y) \rightarrow x+y$ and $(\alpha, x) \rightarrow \alpha x$ are continuous from $X \times X$ and $\mathbb{R} \times X$ to $X$. A topological vector space is called locally convex if there is a basic for the topology consisting of convex sets (that is, sets $A$ such that if $x, y \in A$ then $t x+(1-t) y \in A$ for $0<t<1)$.

Definition 2 (Definition 1.11 in [9]). A Fréchet space is a complete linear metric space or equivalently, a complete total paranormed space. In other words, a locally convex space is called a Fréchet space if it is metrizable and the underlying metric space is complete.

Definition 3 ([5]). Let $\mathcal{M}$ be a class of subsets of a Fréchet space E, we say $\mathcal{M}$ is an admissible set if $\mathfrak{N}_{E} \cap \mathcal{M} \neq \varnothing$ and if $X \in \mathcal{M}$, then $\operatorname{Conv}(X), \bar{X} \in \mathcal{M}$.

Definition 4 ([5]). Let $\mathcal{M}$ be an admissible subset of a Fréchet space E, we say that $\mu: \mathcal{M} \longrightarrow \mathbb{R}_{+}$be a measure of noncompactness on Fréchet space $\mathrm{E}$ if it satisfies the following conditions:

$\left(1^{\circ}\right)$ The family $\operatorname{ker} \mu=\{X \in \mathcal{M}: \mu(X)=0\}$ is nonempty and $k e r \mu \subseteq \mathfrak{N}_{E}$.

$\left(2^{\circ}\right) X \subset Y \Longrightarrow \mu(X) \leq \mu(Y)$.

$\left(3^{\circ}\right) \mu(\bar{X})=\mu(X)$.

(4º) $\mu(\operatorname{Conv} X)=\mu(X)$.

(5) $\mu(\lambda X+(1-\lambda) Y) \leq \lambda \mu(X)+(1-\lambda) \mu(Y)$ for $\lambda \in[0,1]$.

$\left(6^{\circ}\right)$ If $\left\{X_{n}\right\}$ is a sequence of closed sets from $\mathcal{M}$ such that $X_{n+1} \subset X_{n}$ for $n=$ $1,2, \cdots$, and if $\lim _{n \rightarrow \infty} \mu\left(X_{n}\right)=0$, then $X_{\infty}=\cap_{n=1}^{\infty} X_{n} \neq \varnothing$.

Definition 5 (Lim [20]). A function $\varphi$ from $\mathbb{R}_{+}$into itself is called an L-function if $\varphi(0)=0, \varphi(s)>0$ for $s \in(0,+\infty)$, and for every $s \in(0,+\infty)$ there exists $\delta>0$ such that $\varphi(t) \leq s$, for all $t \in[s, s+\delta]$.

Definition 6 ([2]). A function $\theta$ from $\mathbb{R}_{+}$into itself is called a strictly $\mathrm{L}$-function if $\theta(0)=0, \theta(s)>0$ for $s \in(0,+\infty)$, and for every $s \in(0,+\infty)$ there exists $\delta>0$ such that $\theta(t)<s$, for all $t \in[s, s+\delta]$.

\section{FIXED POINT THEOREMS}

In this section, we present some fixed point theorems on a Fréchet space.

Definition 7. Let $\Omega$ be a nonempty subset of a Fréchet space $E, \mathcal{M}$ an admissible set such that $\Omega \in \mathcal{M}$ and $\mu: \mathcal{M} \longrightarrow \mathbb{R}_{+}$be a measure of noncompactness on $E$. We say that an operator $F: \Omega \longrightarrow \Omega$ is a Meir-Keeler condensing operator if for any $\varepsilon>0$, there exists $\delta>0$ such that

$$
\varepsilon \leq \mu(X)<\varepsilon+\delta \Longrightarrow \mu(F X)<\varepsilon,
$$

and $F(X) \in \mathcal{M}$ for any nonempty subset $X \in \mathcal{M}$.

Now, we are ready to state our first main theorem for this section. 
Theorem 4. Let $\Omega$ be a nonempty, closed and convex subset of a Fréchet space $E, \mathcal{M}$ an admissible set such that $\Omega \in \mathcal{M}$ and $\mu: \mathcal{M} \longrightarrow \mathbb{R}_{+}$be a measure of noncompactness on E. Let $F: \Omega \longrightarrow \Omega$ be a continuous and Meir-Keeler condensing operator, then $F$ has at least one fixed point.

Proof. By induction, we obtain a sequence $\left\{\Omega_{n}\right\}$ such that $\Omega_{0}=\Omega$ and $\Omega_{n}=$ $\operatorname{Conv}\left(F \Omega_{n-1}\right), n \geq 1$. It is obvious that $\Omega_{n} \in \mathcal{M}$ and $\Omega_{n+1} \subseteq \Omega_{n}$ for all $n \in \mathbb{N}$. If there exists an integer $N \geq 0$ such that $\mu\left(\Omega_{N}\right)=0$, then $\Omega_{N}$ is compact. Thus, Tychonoff fixed point theorem implies that $F$ has a fixed point. Now assume that $\mu\left(\Omega_{n}\right) \neq 0$ for $n \geq 0$. Define $\varepsilon_{n}=\mu\left(\Omega_{n}\right)$. Since $\Omega_{n+1} \subseteq \Omega_{n}$ and by $\left(2^{\circ}\right)$ so we have $\left\{\varepsilon_{n}\right\}$ is a positive decreasing sequence of real numbers and there exists $\gamma \geq 0$ such that $\varepsilon_{n} \rightarrow \gamma$ as $n \rightarrow \infty$. We claim that $\gamma=0$. Suppose, on the contrary, that $\gamma>0$, then there exists $n_{0}$ such that $n>n_{0}$ implies $\gamma \leq \varepsilon_{n}<\gamma+\delta(\gamma)$, therefore by the definition of Meir-Keeler condensing operator, $\varepsilon_{n+1}<\gamma$ which is a contradiction. Therefore, $\gamma=0$, that is, $\varepsilon_{n} \rightarrow 0$ as $n \rightarrow \infty$. Since the sequence $\left(\Omega_{n}\right)$ is nested, in view of axiom $\left(6^{\circ}\right)$ of Definition 4 we deduce that the set $\Omega_{\infty}=\bigcap_{n=1}^{\infty} \Omega_{n}$ is nonempty, closed and convex subset of the set $\Omega$. Moreover, the set $\Omega_{\infty}$ is invariant under the operator $F$ and belongs to $\operatorname{Ker} \mu$. Thus, applying Tychonoff fixed point theorem, $F$ has a fixed point.

Lemma 1. Let $\varphi: \mathbb{R}_{+} \longrightarrow \mathbb{R}_{+}$be an increasing and right continuous function. Then the following conditions are equivalent:

(a) $\varphi(t)<t$ for any $t>0$ and $\varphi(0)=0$.

(b) $\varphi$ is an L-function.

(c) $\varphi$ is a strictly L-function.

Proof. Let $\varphi$ satisfy condition (a). Since $\varphi(t)<t$ for any $t>0$ and $\varphi$ a right continuous function, so for any $s>0$ there exist $\delta_{s}>0$ such that

$$
|\varphi(t)-\varphi(s)|<s-\varphi(s)
$$

for all $t \in\left[s, s+\delta_{s}\right]$. Thus, $\varphi(t)<s$ for all $t \in\left[s, s+\delta_{s}\right]$ and $\varphi$ satisfies condition (b). Now, assume that $\varphi$ satisfies condition (b). Thus, for any $s>0$ there exists $\delta>0$ such that $\varphi(t) \leq s$ for all $t \in[s, s+\delta]$. Since $\varphi$ is an increasing function, so $\varphi(t)<s$ for all $t \in\left[s, s+\frac{\delta}{2}\right]$ and $\varphi$ satisfies condition (c).

It remains to be shown that condition (c) implies condition (a). Because $\varphi$ is a strictly L-function, it implies that $\varphi(s)<s$ for all $s>0$ and therefore the proof is obvious.

Now, we formulate and prove a fixed point theorem using strictly L-functions as an application of Theorem 4 . 
Theorem 5. Let $\Omega$ be a nonempty, closed and convex subset of a Fréchet space $E$, $\mathcal{M}$ an admissible set such that $\Omega \in \mathcal{M}$ and $\mu: \mathcal{M} \longrightarrow \mathbb{R}_{+}$a measure of noncompactness on $E$. Then $T$ is a Meir-Keeler condensing operator if and only if there exists a (increasing, right continuous) strictly L-function $\vartheta$ such that

$$
\mu(T X) \leq \vartheta(\mu(X)),
$$

for each $X \subseteq \Omega$ and $X \in \mathcal{M}$.

Proof. Let $\varepsilon>0$ be given. By the assumption, there exists $\delta>0$ such that $\vartheta(t)<\varepsilon$ if $\varepsilon \leq t<\varepsilon+\delta$. If $X$ is a subset of $\Omega$ such that

$$
\varepsilon \leq \mu(X)<\varepsilon+\delta(\varepsilon),
$$

thus,

$$
\mu(T(X)) \leq \vartheta(\mu(X))<\varepsilon
$$

and $T$ is a Meir-Keeler condensing operator. For the necessity part, assume that $\mathrm{T}$ is a Meir-Keeler condensing. From the definition of Meir-Keeler condensing, we can define a function $\alpha:(0, \infty) \rightarrow(0, \infty)$, such that

$$
\varepsilon \leq \mu(X)<\varepsilon+2 \alpha(\varepsilon) \Longrightarrow \mu(T(X))<\varepsilon,
$$

for $\varepsilon \in(0, \infty)$. Using such $\alpha$, we define a nondecreasing function $\beta:(0, \infty) \rightarrow$ $[0, \infty)$, by

$$
\beta(t)=\inf \{\varepsilon: t \leq \varepsilon+\alpha(\varepsilon)\}
$$

for $t \in(0, \infty)$. Since $t \leq t+\alpha(t)$, we note that $\beta(t) \leq t$ for $t \in(0, \infty)$. Define a function $\phi_{1}$ from $[0, \infty)$ into itself by

$$
\phi_{1}(t)= \begin{cases}0 & \text { if } t=0, \\ \beta(t) & \text { if } t>0 \text { and } \min \{\varepsilon>0: t \leq \varepsilon+\alpha(\varepsilon)\} \text { exists } \\ \frac{\beta(t)+t}{2} & \text { otherwise. }\end{cases}
$$

Similar to Proposition 1 in [20], $\phi_{1}$ be an L-function. Now, we define $\phi_{2}, \phi_{3}$ and $\vartheta$ from $[0, \infty)$ into itself by

$$
\begin{aligned}
& \phi_{2}(t)=\sup \left\{\phi_{1}(s): s \leq t\right\}, \\
& \phi_{3}(t)=\inf \left\{\phi_{2}(s): s>t\right\}
\end{aligned}
$$

and

$$
\vartheta(t)=\frac{\phi_{3}(t)+t}{2}
$$

for $t \in[0, \infty)$. Then we have

$$
0 \leq \phi_{1}(t) \leq \phi_{2}(t) \leq \phi_{3}(t) \leq \vartheta(t) \leq t
$$

for all $t \in(0, \infty), \phi_{2}$ is a nondecreasing L-function, $\phi_{3}$ is a nondecreasing, right continuous L-function and $\vartheta$ is an increasing, right continuous L-function. Therefore, by Lemma 1 we have $\vartheta$ is a increasing and right continuous strictly L-function. Fix 
$X \in \mathcal{M}$ such that $\mu(X) \neq 0$. From the definition of $\beta$, there exists $\varepsilon \leq \mu(X)$ such that $\varepsilon \leq \mu(X) \leq \varepsilon+\alpha(\varepsilon)$. Thus,

$$
\mu(T X)<\varepsilon \leq \beta(\mu(X)) \leq \vartheta(\mu(X))
$$

holds.

Remark 1. If there exists a strictly L-function $\vartheta$ such that $T$ satisfies in condition (2.2) then there exists an increasing and right continuous strictly L-function $\vartheta^{\prime}$ such that $T$ satisfies in condition (2.2).

\section{INFINITE SYSTEMS OF CONDENSING OPERATORS}

In this section, we prove some existence theorems for a general infinite system of equations involving condensing operators.

Let $\left(E_{i}, d_{i}\right)$ be a Fréchet space for all $i \in \mathbb{N}, d(x, y)=\sup \left\{\frac{1}{2^{i}} \min \left\{1, d_{i}\left(x_{i}, y_{i}\right)\right\}\right.$ : $i \in \mathbb{N}\}, x=\left(x_{1}, x_{2}, \ldots\right), y=\left(y_{1}, y_{2}, \ldots\right) \in \prod_{i \in \mathbb{N}} E_{i}$. Then $\left(\prod_{i \in \mathbb{N}} E_{i}, d\right)$ is a Fréchet space.

Theorem 6 (Tychonoff's theorem[17]). Let $\left\{\left(X_{i}, \tau_{i}\right): i \in \mathbb{N}\right\}$ be any family of topological spaces. Then $\prod_{i \in \mathbb{N}}\left(X_{i}, \tau_{i}\right)$ is compact if and only if each $\left(X_{i}, \tau_{i}\right)$ is compact.

Remark 2. We use the notation $\mathbb{R}^{\omega}$ which denotes the countable Cartesian product of $\mathbb{R}_{+}$with itself, and $l^{\infty}$ consists of all bounded sequences of scalars.

Now we are ready to state and prove the main results of this section.

Theorem 7. Suppose $\mu_{i}$ be a measure of noncompactness on Fréchet spaces $E_{i}$ for all $i \in \mathbb{N}$. Moreover assume that the function $F: l^{\infty} \longrightarrow \mathbb{R}_{+}$is convex, nondecreasing and $F\left(\left(x_{i}\right)_{i=1}^{\infty}\right)=0$ if and only if $x_{i}=0$ for all $i \in \mathbb{N}$. If we define

$$
\mathcal{M}=\left\{X \subseteq \prod_{i=1}^{\infty} E_{i}: \sup _{i}\left\{\mu_{i}\left(\pi_{i}(X)\right)\right\}<\infty\right\},
$$

where $\pi_{i}(X)$ denotes the natural projections of $\prod_{i=1}^{\infty} E_{i}$ into $E_{i}$ and $\mu: \mathcal{M} \longrightarrow \mathbb{R}_{+}$be defined by

$$
\mu(X)=F\left(\left(\mu_{i}\left(\pi_{i}(X)\right)\right)_{i=1}^{\infty}\right),
$$

then $\mathcal{M}$ is an admissible set and $\mu$ is a measure of noncompactness on Fréchet space $E=\prod_{i=1}^{\infty} E_{i}$ 
Proof. It can be easily verified that $\mathcal{M}$ is an admissible set. Now, we prove $\mu$ is a measure of noncompactness on $E=\prod_{i=1}^{\infty} E_{i}$. For proving the condition $\left(2^{\circ}\right)$, suppose that $X \subset Y$. Since $\mu_{i}$ be a measure of noncompactness, we have $\mu_{i}\left(\pi_{i}(X)\right) \leq$ $\mu_{i}\left(\pi_{i}(Y)\right)$ for all $i \in \mathbb{N}$, and using $F$ is nondecreasing we imply that

$$
\mu(X)=F\left(\left(\mu_{i}\left(\pi_{i}(X)\right)\right)_{i=1}^{\infty}\right) \leq \mu(Y)=F\left(\left(\mu_{i}\left(\pi_{i}(Y)\right)\right)_{i=1}^{\infty}\right),
$$

which shows that the condition $\left(2^{\circ}\right)$ is valid. The properties $\left(3^{\circ}\right)-\left(5^{\circ}\right)$ are simple consequences of

$$
\begin{aligned}
& \pi_{i}(\lambda U+(1-\lambda) V)=\lambda \pi_{i}(U)+(1-\lambda) \pi_{i}(V), \\
& \pi_{i}(\operatorname{Conv} X)=\operatorname{Conv} \pi_{i}(X), \\
& \pi_{i}(X) \subseteq \pi_{i}(\bar{X}) \subseteq \overline{\pi_{i}(X)} .
\end{aligned}
$$

Now we show $\left(1^{\circ}\right)$. If $\mu(X)=0$ for $X \in \mathcal{M}$ then $\mu_{i}\left(\pi_{i}(X)\right)=0$ for each $i \in \mathbb{N}$. Hence, by virtue of $\left(1^{\circ}\right)$ of Definition 4 for measure of noncompactness $\mu_{i}, \pi_{i}(X)$ is relatively compact for all $i \in \mathbb{N}$ and by Theorem $6, \prod_{i=1}^{\infty} \pi_{i}(X)$ is relatively compact. Thus, $X \subseteq \prod_{i=1}^{\infty} \pi_{i}(X)$ is relatively compact. Finally, it suffices to show $\left(6^{\circ}\right)$. suppose that $\left\{X_{n}\right\}$ is a sequence of closed sets from $\mathcal{M}$ such that $X_{n+1} \subseteq X_{n}$ for $n \in \mathbb{N}$ and $\lim _{n \rightarrow \infty} \mu\left(X_{n}\right)=0$. So we have

$$
\lim _{n \rightarrow \infty} F\left(\left(\mu_{i}\left(\pi_{i}\left(X_{n}\right)\right)\right)_{i=1}^{\infty}\right)=0 .
$$

Since $X_{n+1} \subseteq X_{n}, \mu_{i}\left(\pi_{i}\left(X_{n+1}\right)\right) \leq \mu_{i}\left(\pi_{i}\left(X_{n}\right)\right)$ and $\mu_{i}\left(\pi_{i}\left(X_{n}\right)\right) \geq 0$ for all $i \in \mathbb{N}$. Thus, there is an $r_{i} \geq 0$ so that

$$
\lim _{n \rightarrow \infty} \mu_{i}\left(\pi_{i}\left(X_{n}\right)\right)=r_{i} .
$$

Because $F$ is continuous, then

$$
\lim _{n \rightarrow \infty} F\left(\left(\mu_{i}\left(\pi_{i}\left(X_{n}\right)\right)\right)_{i=1}^{\infty}\right)=F\left(\lim _{n \rightarrow \infty}\left(\mu_{i}\left(\pi_{i}\left(X_{n}\right)\right)\right)_{i=1}^{\infty}\right)=F\left(\left(r_{i}\right)_{i=1}^{\infty}\right) .
$$

On the other hand, using (3.3) we have $F\left(\left(r_{i}\right)_{i=1}^{\infty}\right)=0$. By assumption of the theorem we imply that $r_{i}=0$ for all $i \in \mathbb{N}$, and so $\lim _{n \rightarrow \infty} \mu_{i}\left(\pi_{i}\left(X_{n}\right)\right)=0$. By $\left(6^{\circ}\right)$ of definition of measure of noncompactness on $E_{n}$ we have $X_{i}^{\infty}=\bigcap_{n=1}^{\infty} \pi_{i}\left(X_{n}\right) \neq \varnothing$ for all $i \in \mathbb{N}$. Therefore we get $\prod_{i=1}^{\infty} X_{i}^{\infty} \subseteq X_{\infty}$ and $X_{\infty} \neq \varnothing$. This completes the proof. 
Example 1. Let $\mu_{n}(n \in \mathbb{N})$ be measures of noncompactness on Fréchet spaces $E_{n}$, respectively. Considering $F_{1}\left(\left(x_{n}\right)_{n=1}^{\infty}\right)=\sup _{n \in \mathbb{N}} b_{n} x_{n}$ and $F_{2}\left(\left(x_{n}\right)_{n=1}^{\infty}\right)=\sum_{n=1}^{\infty} a_{n} x_{n}$ (the functions $F 1, F 2$ are defined on $l^{\infty}$ ) such that $a_{n}, b_{n} \in \mathbb{R}_{+}, \sum_{n=1}^{\infty} a_{n}<\infty$ and $\left\{b_{n}\right\}$ be a bounded sequence, then all the conditions of Theorem 7 are satisfied. Therefore, $\widetilde{\mu}_{1}:=\sup _{n \in \mathbb{N}} b_{n} \mu\left(X_{n}\right)$ and $\left.\widetilde{\mu}_{2}:=\sum_{n=1}^{\infty} a_{n} \mu\left(X_{n}\right)\right)$ define measures of noncompactness in the Fréchet space $E=\prod_{i=1}^{\infty} E_{i}$ where $X_{n}(n \in \mathbb{N})$, denotes the natural projections of X into $E_{n}$.

Theorem 8. Let $\Omega_{i}(i \in \mathbb{N})$ be a nonempty, convex and closed subset of a Fréchet space $E_{i}, \mu_{i}$ an arbitrary measure of noncompactness on $E_{i}$ and $\sup _{i}\left\{\mu_{i}\left(\Omega_{i}\right)\right\}<\infty$. Let $F_{i}: \prod_{i=1}^{\infty} \Omega_{i} \longrightarrow \Omega_{i}(i=1,2, \ldots)$ be a continuous operator such that

$$
a_{i} \mu_{i}\left(F_{i}\left(\prod_{j=1}^{\infty} X_{j}\right)\right) \leq \varphi\left(\sup _{j}\left\{a_{j} \mu_{j}\left(X_{j}\right)\right\}\right)
$$

for any subset $X_{i}$ of $\Omega_{i}(i \in \mathbb{N})$ where $\varphi: \mathbb{R}_{+} \longrightarrow \mathbb{R}_{+}$is a strictly L-function and $\left\{a_{i}\right\}$ is a bounded sequence of positive real numbers. Then there exist $\left(x_{j}^{*}\right)_{j=1}^{\infty} \in \prod_{j=1}^{\infty} \Omega_{j}$ such that

$$
F_{i}\left(\left(x_{j}^{*}\right)_{j=1}^{\infty}\right)=x_{i}^{*}
$$

for all $i \in \mathbb{N}$.

Proof. Let us consider $\widetilde{F}: \prod_{i=1}^{\infty} \Omega_{i} \longrightarrow \prod_{i=1}^{\infty} \Omega_{i}$ in the following way:

$$
\widetilde{F}\left(\left(x_{j}\right)_{j=1}^{\infty}\right)=\left(F_{1}\left(\left(x_{j}\right)_{j=1}^{\infty}\right), F_{2}\left(\left(x_{j}\right)_{j=1}^{\infty}\right), \ldots, F_{i}\left(\left(x_{j}\right)_{j=1}^{\infty}\right), \ldots\right)
$$

for all $\left(x_{j}\right)_{j=1}^{\infty} \in \prod_{i=1}^{\infty} \Omega_{i}$. It is obvious that $\widetilde{F}$ is continuous. It suffices to show that the hypothesis (2.2) of Theorem 5 holds where $\mu$ is defined by Example 1. Take an 
arbitrary nonempty subset $X$ of $\prod_{i=1}^{\infty} \Omega_{i}$. Now, by (2) and (3.4) we obtain

$$
\begin{aligned}
\mu(\widetilde{F}(X)) & \leq \mu\left(\prod_{i=1}^{\infty} F_{i}\left(\prod_{j=1}^{\infty} \pi_{j}(X)\right)\right) \\
& =\sup _{i} a_{i} \mu_{i}\left(F_{i}\left(\left(\prod_{j=1}^{\infty} \pi_{j}(X)\right)\right)\right) \\
& \leq \sup _{i} \varphi\left(\sup _{j} a_{j} \mu_{j}\left(\pi_{j}(X)\right)\right) \\
& \leq \sup _{i} \varphi(\mu(X)) \\
& \leq \varphi(\mu(X)) .
\end{aligned}
$$

Thus, using Theorem 5, $\widetilde{F}$ is a continuous and Meir-Keeler condensing operator. Now applying Theorem $4, \widetilde{F}$ has a fixed point and there exist $\left(x_{j}^{*}\right)_{j=1}^{\infty} \in \prod_{j=1}^{\infty} \Omega_{j}$ such that

$$
\left(x_{j}^{*}\right)_{j=1}^{\infty}=\widetilde{F}\left(\left(x_{j}^{*}\right)_{j=1}^{\infty}\right)=\left(F_{1}\left(\left(x_{j}^{*}\right)_{j=1}^{\infty}\right), F_{2}\left(\left(x_{j}^{*}\right)_{j=1}^{\infty}\right), \ldots, F_{j}\left(\left(x_{j}^{*}\right)_{j=1}^{\infty}\right), \ldots\right)
$$

and that (3.5) holds.

\section{EXISTENCE OF A SOLUTION FOR A INFINITE SYSTEM OF INTEGRAL EQUATIONS}

In the following section, we will work in the classical Banach space $B C\left(\mathbb{R}_{+} \times\right.$ $\mathbb{R}_{+}$) consisting of all real functions defined, bounded and continuous on $\mathbb{R}_{+} \times \mathbb{R}_{+}$ equipped with the standard norm

$$
\|x\|=\sup \{|x(t, s)|: t, s \geq 0\} .
$$

Now, we present the definition of a special measure of noncompactness in $B C\left(\mathbb{R}_{+} \times\right.$ $\mathbb{R}_{+}$) which will be needed in the sequel.

To do this, let $X$ be a fixed nonempty and bounded subset of $B C\left(\mathbb{R}_{+} \times \mathbb{R}_{+}\right)$and fix a positive number $T$. For $x \in X$ and $\epsilon>0$, denote by $\omega^{T}(x, \epsilon)$ the modulus of the continuity of function $x$ on the interval $[0, T]$, i.e.,

$$
\omega^{T}(x, \epsilon)=\sup \{|x(t, s)-x(u, v)|: t, s, u, v \in[0, T],|t-u| \leq \epsilon,|s-v| \leq \epsilon\} .
$$

Further, let us put

$$
\begin{gathered}
\omega^{T}(X, \epsilon)=\sup \left\{\omega^{T}(x, \epsilon): x \in X\right\}, \\
\omega_{0}^{T}(X)=\lim _{\epsilon \rightarrow 0} \omega^{T}(X, \epsilon)
\end{gathered}
$$


and

$$
\omega_{0}(X)=\lim _{T \rightarrow \infty} \omega_{0}^{T}(X) .
$$

Moreover, for two fixed numbers $t, s \in \mathbb{R}_{+}$let us the define the function $\mu$ on the family $\mathfrak{M}_{B C\left(\mathbb{R}_{+} \times \mathbb{R}_{+}\right)}$by the following formula

$$
\mu(X)=\omega_{0}(X)+\limsup _{\|(t, s)\| \rightarrow \infty} \operatorname{diam} X(t, s),
$$

where $\|(t, s)\|=\max (t, s)$ and $X(t, s)=\{x(t, s): x \in X\}$. Similar to [8], it can be shown that the function $\mu$ is a measure of noncompactness in the space $B C\left(\mathbb{R}_{+} \times\right.$ $\mathbb{R}_{+}$).

As an application of Theorem 8 we prove the existence of solutions for the infinite system of integral equations of Hammerstein type in two variables (1.2).

Theorem 9. Assume that the following conditions are satisfied:

(a 1$) f_{n}: \mathbb{R}_{+} \times \mathbb{R}_{+} \times \mathbb{R}^{n} \times \mathbb{R} \longrightarrow \mathbb{R}(n \in \mathbb{N})$ is continuous. Moreover, there exists a nondecreasing, right continuity and concave strictly $L$-function $\varphi$ such that

$$
\left|f_{n}\left(t, s, x_{1}, \ldots, x_{n}, u\right)-f_{n}\left(t, s, y_{1}, \ldots, y_{n}, v\right)\right| \leq \varphi\left(\max _{1 \leq i \leq n}\left|x_{i}-y_{i}\right|\right)+|u-v|
$$

$\left(a_{2}\right) \quad M:=\sup \left\{\left|f_{n}(t, s, 0, \ldots, 0)\right|: t, s \in \mathbb{R}_{+}, n \in \mathbb{N}\right\}<\infty ;$

$\left(a_{3}\right) k_{n}: \mathbb{R}_{+} \times \mathbb{R}_{+} \times \mathbb{R}_{+} \times \mathbb{R}_{+} \longrightarrow \mathbb{R}$ are continuous functions for all $n \in \mathbb{N}$;

$\left(a_{4}\right) h_{n}: \mathbb{R}_{+} \times \mathbb{R}_{+} \times \mathbb{R}^{\omega} \longrightarrow \mathbb{R}(n \in \mathbb{N})$ is continuous and there exist a continuous function $a_{n}: \mathbb{R}_{+} \times \mathbb{R}_{+} \longrightarrow \mathbb{R}_{+}$and a continuous and nondecreasing function $b_{n}: \mathbb{R}_{+} \longrightarrow \mathbb{R}_{+}$such that

$$
\left|h_{n}\left(t, s,\left(x_{j}\right)_{j=1}^{\infty}\right)\right| \leq a_{n}(t, s) b_{n}\left(\sup _{1 \leq j<\infty}\left|x_{j}\right|\right)
$$

for all $t, s \in \mathbb{R}_{+}$and $\left(x_{j}\right)_{j=1}^{\infty} \in \mathbb{R}^{\omega}$ with $\sup _{1 \leq j<\infty}\left|x_{j}\right|<\infty$. Also the function $(v, w) \longrightarrow a_{n}(v, w) k_{n}(v, w, t, s)$ is integrable over $\mathbb{R}_{+} \times \mathbb{R}_{+}$for any fixed $t, s \in \mathbb{R}_{+}$and $n \in \mathbb{N}$.

$\left(a_{5}\right)$ there exists a positive constant $D$ such that

$$
D=\sup \left\{\int_{0}^{\infty} \int_{0}^{\infty} a_{n}(v, w)\left|k_{n}(t, s, v, w)\right| d v d w: t, s \in \mathbb{R}_{+}, n \in \mathbb{N}\right\}<\infty,
$$

$$
\lim _{\|t, s\| \rightarrow \infty} \int_{0}^{\infty} \int_{0}^{\infty} a_{n}(v, w)\left|k_{n}(t, s, v, w)\right| d v d w=0
$$

$\left(_{6}\right)$ the following equalities are hold:

$$
\begin{aligned}
& \lim _{T \rightarrow \infty}\left\{\sup \left\{\int_{T}^{\infty} \int_{0}^{T} a_{n}(v, w)\left|k_{n}(t, s, v, w)\right| d v d w: t, s \in \mathbb{R}_{+}\right\}\right\}=0, \\
& \lim _{T \rightarrow \infty}\left\{\sup \left\{\int_{0}^{\infty} \int_{T}^{\infty} a_{n}(v, w)\left|k_{n}(t, s, v, w)\right| d v d w: t, s \in \mathbb{R}_{+}\right\}\right\}=0,
\end{aligned}
$$


for all $n \in \mathbb{N}$;

$\left(a_{7}\right)$ there exists a positive solution $r_{0}$ of the inequality

$$
\varphi(r)+M+D b_{n}(r) \leq r
$$

for all $n \in \mathbb{N}$.

Then the infinite system of equations (1.2) has at least one solution in the space $\left(B C\left(\mathbb{R}_{+} \times \mathbb{R}_{+}\right)\right)^{\omega}$.

Proof. Let us fix arbitrarily $n \in \mathbb{N}$. Define $H_{n}:\left(B C\left(\mathbb{R}_{+} \times \mathbb{R}_{+}\right)\right)^{\omega} \longrightarrow B C\left(\mathbb{R}_{+} \times\right.$ $\left.\mathbb{R}_{+}\right)$by

$$
\begin{aligned}
H_{n}\left(\left(x_{j}\right)_{j=1}^{\infty}\right)(t, s)= & f_{n}\left(t, s, x_{1}(t, s) \ldots, x_{n}(t, s),\right. \\
& \left.\int_{0}^{\infty} \int_{0}^{\infty} k_{n}(t, s, v, w) h_{n}\left(v, w,\left(x_{j}(v, w)\right)_{j=1}^{\infty}\right) d v d w\right) .
\end{aligned}
$$

In light of (4.4) and assumptions $\left(a_{1}\right)-\left(a_{7}\right), f_{n}$ is continuous, $k_{n}$ is continuous, $h_{n}$ is continuous and $x_{i}$ for $i \in \mathbb{N}$ are continuous. On the other hand, integral of continuous function is continuous. Therefore, we infer that the function $H_{n}\left(\left(x_{j}\right)_{j=1}^{\infty}\right)$ is continuous for arbitrarily $\left(x_{j}\right)_{j=1}^{\infty} \in\left(B C\left(\mathbb{R}_{+} \times \mathbb{R}_{+}\right)\right)^{\omega}$ because it is the composition of continuous functions. Moreover, in view of our assumptions, for arbitrarily fixed $\left(x_{j}\right)_{j=1}^{\infty} \in\left(B C\left(\mathbb{R}_{+} \times \mathbb{R}_{+}\right)\right)^{\omega}$ and $t, s \in \mathbb{R}_{+}$, we obtain

$$
\begin{aligned}
& \left|H_{n}\left(\left(x_{j}\right)_{j=1}^{\infty}\right)(t, s)\right| \\
\leq & \mid f_{n}\left(t, s, x_{1}(t, s) \ldots, x_{n}(t, s), \int_{0}^{\infty} \int_{0}^{\infty} k_{n}(t, s, v, w) h_{n}\left(v, w,\left(x_{j}(v, w)\right)_{j=1}^{\infty}\right) d v d w\right) \\
& -f_{n}(t, s, 0, \ldots, 0)|+| f_{n}(t, s, 0, \ldots, 0) \mid \\
\leq & \varphi\left(\max _{1 \leq i \leq n}\left|x_{i}(t, s)\right|\right)+\left|f_{n}(t, s, 0, \ldots, 0)\right| \\
& +\int_{0}^{\infty} \int_{0}^{\infty}\left|k_{n}(t, s, v, w)\right|\left|a_{n}(v, w)\right| b_{n}\left(\left|\left(x_{j}(v, w)\right)_{j=1}^{\infty}\right|\right) \\
\leq & \varphi\left(\max _{1 \leq i \leq n}\left|x_{i}(t, s)\right|\right)+M+D b_{n}\left(\sup _{1 \leq j<\infty}\left|x_{j}(v, w)\right|\right) \\
\leq & \varphi\left(\max _{1 \leq i \leq n}\left\|x_{i}\right\|\right)+M+D b_{n}\left(\sup _{1 \leq j<\infty}\left\|x_{j}\right\|\right) .
\end{aligned}
$$

Thus,

$$
\left\|H_{n}(x)\right\| \leq \varphi\left(\max _{1 \leq i \leq n}\left\|x_{i}\right\|\right)+M+D b_{n}\left(\sup _{1 \leq j<\infty}\left\|x_{j}\right\|\right)
$$

and $H_{n}\left(\left(x_{j}\right)_{j=1}^{\infty}\right) \in B C\left(\mathbb{R}_{+} \times \mathbb{R}_{+}\right)$for any $\left(x_{j}\right)_{j=1}^{\infty} \in\left(B C\left(\mathbb{R}_{+} \times \mathbb{R}_{+}\right)\right)^{\omega}$. By (4.6) and using $\left(a_{7}\right)$, the function $H_{n}$ maps $\left(\bar{B}_{r_{0}}\right)^{\omega}$ into $\bar{B}_{r_{0}}$.

Now we claim that $H_{n}$ is a continuous function on $\left(\bar{B}_{r_{0}}\right)^{\omega}$ for all $n \in \mathbb{N}$. To do this, let us fix $0<\varepsilon<\frac{1}{2^{n}}$ and take arbitrary $x=\left(\left(x_{j}\right)_{j=1}^{\infty}\right), y=\left(\left(y_{j}\right)_{j=1}^{\infty}\right) \in\left(B C\left(\mathbb{R}_{+} \times\right.\right.$ 
$\left.\left.\mathbb{R}_{+}\right)\right)^{\omega}$ such that $d(x, y)=\sup \left\{\frac{1}{2^{i}} \min \left\{1,\left\|x_{i}-y_{i}\right\|\right\}: i \in \mathbb{N}\right\}<\varepsilon$. Then, for $t, s, v, w \in$ $\mathbb{R}_{+}$, we get

$$
\begin{aligned}
\mid H_{n} & \left(\left(x_{j}\right)_{j=1}^{\infty}\right)(t, s)-H_{n}\left(\left(y_{j}\right)_{j=1}^{\infty}\right)(t, s) \mid \\
\leq & \mid f_{n}\left(t, s, x_{1}(t, s), \ldots, x_{n}(t, s), \int_{0}^{\infty} \int_{0}^{\infty} k_{n}(t, s, v, w) h_{n}\left(v, w,\left(x_{j}(v, w)\right)_{j=1}^{\infty}\right) d v d w\right) \\
& -f_{n}\left(t, s, y_{1}(t, s), \ldots, y_{n}(t, s), \int_{0}^{\infty} \int_{0}^{\infty} k_{n}(t, s, v, w) h_{n}\left(v, w,\left(y_{j}(v, w)\right)_{j=1}^{\infty}\right) d v d w\right) \mid \\
\leq & \varphi\left(\max _{1 \leq i \leq n}\left|x_{i}(t, s)-y_{i}(t, s)\right|\right) \\
& +\left|\int_{0}^{\infty} \int_{0}^{\infty} k_{n}(t, s, v, w)\left[h_{n}\left(v, w,\left(x_{j}(v, w)\right)_{j=1}^{\infty}\right)-h_{n}\left(v, w,\left(y_{j}(v, w)\right)_{j=1}^{\infty}\right)\right] d v d w\right| .
\end{aligned}
$$

On the other hand, assumption $\left(a_{5}\right)$ ensure that there exists a positive number $T$ such that for $\max (t, s)>T$ we have

$$
\begin{aligned}
\left|H_{n}\left(\left(x_{j}\right)_{j=1}^{\infty}\right)(t, s)-H_{n}\left(\left(y_{j}\right)_{j=1}^{\infty}\right)(t, s)\right| \leq & \varphi\left(\max _{1 \leq i \leq n}\left|x_{i}(t, s)-y_{i}(t, s)\right|\right) \\
& +2 b_{n}\left(r_{0}\right) \int_{0}^{\infty} \int_{0}^{\infty}\left|k_{n}(t, s, v, w)\right| a_{n}(v, w) d v d w \\
\leq & \varphi\left(2^{n} \varepsilon\right)+b_{n}\left(r_{0}\right) \varepsilon .
\end{aligned}
$$

Suppose that $t, s \in[0, T]$. By applying the assumptions, we infer that

$$
\begin{aligned}
\mid & H_{n}\left(\left(x_{j}\right)_{j=1}^{\infty}\right)(t, s)-H_{n}\left(\left(y_{j}\right)_{j=1}^{\infty}\right)(t, s) \mid \\
\leq & \varphi\left(\max _{1 \leq i \leq n}\left|x_{i}(t, s)-y_{i}(t, s)\right|\right) \\
& +\left|\int_{0}^{\infty} \int_{0}^{\infty} k_{n}(t, s, v, w)\left[h_{n}\left(v, w,\left(x_{j}(v, w)\right)_{j=1}^{\infty}\right)-h_{n}\left(v, w,\left(y_{j}(v, w)\right)_{j=1}^{\infty}\right)\right] d v d w\right| \\
\leq & \varphi\left(2^{n} \varepsilon\right)+\left\{\int _ { 0 } ^ { \infty } \left\{\int_{0}^{T}\left|k_{n}(t, s, v, w)\right|\left|h_{n}\left(v, w,\left(x_{j}(v, w)\right)_{j=1}^{\infty}\right)-h_{n}\left(v, w,\left(y_{j}(v, w)\right)_{j=1}^{\infty}\right)\right| d v\right.\right. \\
& \left.\left.+\int_{T}^{\infty}\left|k_{n}(t, s, v, w)\right|\left[\left|h_{n}\left(v, w,\left(x_{j}(v, w)\right)_{j=1}^{\infty}\right)\right|+h_{n}\left(v, w,\left(y_{j}(v, w)\right)_{j=1}^{\infty}\right) \mid\right] d v\right\} d w\right\} \\
\leq & \varphi\left(2^{n} \varepsilon\right)+\int_{0}^{T} \int_{0}^{T}\left|k_{n}(t, s, v, w)\right|\left|h_{n}\left(v, w,\left(x_{j}(v, w)\right)_{j=1}^{\infty}\right)-h_{n}\left(v, w,\left(y_{j}(v, w)\right)_{j=1}^{\infty}\right)\right| d v d w \\
& +\int_{0}^{T} \int_{T}^{\infty}\left|k_{n}(t, s, v, w)\right|\left[\left|h_{n}\left(v, w,\left(x_{j}(v, w)\right)_{j=1}^{\infty}\right)\right|+\left|h_{n}\left(v, w,\left(y_{j}(v, w)\right)_{j=1}^{\infty}\right)\right|\right] d v d w \\
& +\int_{T}^{\infty} \int_{0}^{\infty}\left|k_{n}(t, s, v, w)\right|\left[\left|h_{n}\left(v, w,\left(x_{j}(v, w)\right)_{j=1}^{\infty}\right)\right|+\left|h_{n}\left(v, w,\left(y_{j}(v, w)\right)_{j=1}^{\infty}\right)\right|\right] d v d w \\
\leq & \varphi\left(2^{n} \varepsilon\right)+K_{T}^{n} \omega_{r_{0}}^{T}\left(h_{n}, \varepsilon\right)+2 b_{n}\left(r_{0}\right) \int_{0}^{T} \int_{T}^{\infty} a_{n}(v, w)\left|k_{n}(t, s, v, w)\right| d v d w \\
& +2 b_{n}\left(r_{0}\right) \int_{T}^{\infty} \int_{0}^{\infty} a_{n}(v, w)\left|k_{n}(t, s, v, w)\right| d v d w,
\end{aligned}
$$

where

$$
K_{T}^{n}=\sup \left\{k_{n}(t, s, v, w): t, s, v, w \in[0, T]\right\}
$$




$$
\begin{aligned}
& \omega_{r_{0}}^{T}\left(h_{n}, \varepsilon\right)=\sup \left\{\left|h_{n}\left(v, w,\left(x_{j}\right)_{j=1}^{\infty}\right)-h_{n}\left(v, w,\left(y_{j}\right)_{j=1}^{\infty}\right)\right|\right. \\
& \left.: v, w \in[0, T], x_{i}, y_{i} \in\left[-r_{0}, r_{0}\right],\left|x_{i}-y_{i}\right| \leq \varepsilon\right\} .
\end{aligned}
$$

From the continuity of the function $h_{n}$ on the compact set $[0, T] \times[0, T] \times\left[-r_{0}, r_{0}\right]^{\omega}$ (by using Tychonoff's theorem), we have $\omega_{r_{0}}^{T}\left(h_{n}, \varepsilon\right) \longrightarrow 0$ as $\varepsilon \longrightarrow 0$ and in view of assumption $\left(a_{6}\right)$ we can choose $T$ in such a way that three last terms of the above estimate are sufficiently small. Thus $H_{n}$ is a continuous function on $\left(B C\left(\mathbb{R}_{+} \times\right.\right.$ $\left.\left.\mathbb{R}_{+}\right)\right)^{\omega}$.

Now we assert that $H_{n}$ satisfies all the conditions of Theorem 8. Let $X_{i}$ be nonempty and bounded subsets of $\bar{B}_{r_{0}}$ for all $i \in \mathbb{N}$ such that $\sup _{i}\left(\mu\left(X_{i}\right)\right)<\infty$, and assume that $T>0$ and $\varepsilon>0$ are arbitrary constants. Let $t_{1}, t_{2}, s_{1}, s_{2} \in[0, T]$ such that $\mid t_{2}-$ $t_{1}|\leq \varepsilon,| s_{2}-s_{1} \mid \leq \varepsilon$ and $x_{i} \in X_{i}$ for all $i \in \mathbb{N}$. Then, by the assumptions we have:

$$
\begin{aligned}
& \left|H_{n}\left(\left(x_{i}\right)_{i=1}^{\infty}\right)\left(t_{2}, s_{2}\right)-H_{n}\left(\left(x_{i}\right)_{i=1}^{\infty}\right)\left(t_{1}, s_{1}\right)\right| \\
& \leq \mid f_{n}\left(t_{2}, s_{2}, x_{1}\left(t_{2}, s_{2}\right), \ldots, x_{n}\left(t_{2}, s_{2}\right), \int_{0}^{\infty} \int_{0}^{\infty} k_{n}\left(t_{2}, s_{2}, v, w\right) h_{n}\left(v, w,\left(x_{i}(v, w)\right)_{i=1}^{\infty}\right) d v d w\right) \\
& -f_{n}\left(t_{1}, s_{1}, x_{1}\left(t_{1}, s_{1}\right), \ldots, x_{n}\left(t_{1}, s_{1}\right), \int_{0}^{\infty} \int_{0}^{\infty} k_{n}\left(t_{1}, s_{1}, v, w\right) h_{n}\left(v, w,\left(x_{i}(v, w)\right)_{i=1}^{\infty}\right) d v d w\right) \\
& \leq \mid f_{n}\left(t_{2}, s_{2}, x_{1}\left(t_{2}, s_{2}\right), \ldots, x_{n}\left(t_{2}, s_{2}\right), \int_{0}^{\infty} \int_{0}^{\infty} k_{n}\left(t_{2}, s_{2}, v, w\right) h_{n}\left(v, w,\left(x_{i}(v, w)\right)_{j=1}^{\infty}\right) d v d w\right) \\
& -f_{n}\left(t_{2}, s_{2}, x_{1}\left(t_{1}, s_{1}\right), \ldots, x_{n}\left(t_{1}, s_{1}\right), \int_{0}^{\infty} \int_{0}^{\infty} k_{n}\left(t_{2}, s_{2}, v, w\right) h_{n}\left(v, w,\left(x_{i}(v, w)\right)_{i=1}^{\infty}\right) d v d w\right) \\
& +\mid f_{n}\left(t_{2}, s_{2}, x_{1}\left(t_{1}, s_{1}\right), \ldots, x_{n}\left(t_{1}, s_{1}\right), \int_{0}^{\infty} \int_{0}^{\infty} k_{n}\left(t_{2}, s_{2}, v, w\right) h_{n}\left(v, w,\left(x_{i}(v, w)\right)_{i=1}^{\infty}\right) d v d w\right) \\
& -f_{n}\left(t_{1}, s_{1}, x_{1}\left(t_{1}, s_{1}\right), \ldots, x_{n}\left(t_{1}, s_{1}\right), \int_{0}^{\infty} \int_{0}^{\infty} k_{n}\left(t_{2}, s_{2}, v, w\right) h_{n}\left(v, w,\left(x_{i}(v, w)\right)_{i=1}^{\infty}\right) d v d w\right) \\
& +\mid f_{n}\left(t_{1}, s_{1}, x_{1}\left(t_{1}, s_{1}\right), \ldots, x_{n}\left(t_{1}, s_{1}\right), \int_{0}^{\infty} \int_{0}^{\infty} k_{n}\left(t_{2}, s_{2}, v, w\right) h_{n}\left(v, w,\left(x_{i}(v, w)\right)_{i=1}^{\infty}\right) d v d w\right) \\
& -f_{n}\left(t_{1}, s_{1}, x_{1}\left(t_{1}, s_{1}\right), \ldots, x_{n}\left(t_{1}, s_{1}\right), \int_{0}^{\infty} \int_{0}^{\infty} k_{n}\left(t_{1}, s_{1}, v, w\right) h_{n}\left(v, w,\left(x_{i}(v, w)\right)_{i=1}^{\infty}\right) d v d w\right) \\
& \leq \varphi\left(\max _{1 \leq j \leq n}\left|x_{j}\left(t_{2}, s_{2}\right)-x_{j}\left(t_{1}, s_{1}\right)\right|\right)+\omega_{r_{0}, D_{1}}^{T}\left(f_{n}, \varepsilon\right) \\
& +\left|\int_{0}^{\infty} \int_{0}^{\infty}\left[k_{n}\left(t_{2}, s_{2}, v, w\right)-k_{n}\left(t_{1}, s_{1}, v, w\right)\right] h_{n}\left(v, w,\left(x_{i}(v, w)\right)_{i=1}^{\infty}\right) d v d w\right| \\
& \leq \varphi\left(\max _{1 \leq j \leq n}\left|x_{j}\left(t_{2}, s_{2}\right)-x_{j}\left(t_{1}, s_{1}\right)\right|\right)+\omega_{r_{0}, D_{1}}^{T}\left(f_{n}, \varepsilon\right) \\
& +\int_{0}^{T} \int_{0}^{T}\left|k_{n}\left(t_{2}, s_{2}, v, w\right)-k_{n}\left(t_{1}, s_{1}, v, w\right)\right|\left|h_{n}\left(v, w,\left(x_{i}(v, w)\right)_{i=1}^{\infty}\right)\right| d v d w \\
& +\int_{0}^{T} \int_{T}^{\infty}\left|k_{n}\left(t_{2}, s_{2}, v, w\right)-k_{n}\left(t_{1}, s_{1}, v, w\right)\right|\left|h_{n}\left(v, w,\left(x_{i}(v, w)\right)_{i=1}^{\infty}\right)\right| d v d w \\
& +\int_{T}^{\infty} \int_{0}^{\infty}\left|k_{n}\left(t_{2}, s_{2}, v, w\right)-k_{n}\left(t_{1}, s_{1}, v, w\right)\right|\left|h_{n}\left(v, w,\left(x_{i}(v, w)\right)_{i=1}^{\infty}\right)\right| d v d w
\end{aligned}
$$




$$
\begin{aligned}
\leq & \varphi\left(\max _{1 \leq j \leq n} \omega^{T}\left(x_{j}, \varepsilon\right)\right)+\omega_{r_{0}, D_{1}}^{T}\left(f_{n}, \varepsilon\right)+T^{2} U_{r_{0}}^{T} \omega^{T}\left(k_{n}, \varepsilon\right) \\
& +b_{n}\left(r_{0}\right) \int_{0}^{T} \int_{T}^{\infty}\left[\left|k_{n}\left(t_{2}, s_{2}, v, w\right)\right|+\left|k_{n}\left(t_{1}, s_{1}, v, w\right)\right|\right] a_{n}(v, w) d v d w \\
& +b_{n}\left(r_{0}\right) \int_{T}^{\infty} \int_{0}^{\infty}\left[\left|k_{n}\left(t_{2}, s_{2}, v, w\right)\right|+\left|k_{n}\left(t_{1}, s_{1}, v, w\right)\right|\right] a_{n}(v, w) d v d w
\end{aligned}
$$

where

$$
\begin{aligned}
& D_{1}=b_{n}\left(r_{0}\right) D\left(\text { see assumption }\left(a_{5}\right)\right) \\
& \omega_{r_{0}, D_{1}}^{T}\left(f_{n}, \varepsilon\right)=\sup \left\{\left|f_{n}\left(t_{2}, s_{2}, x_{1}, \cdots, x_{n}, y\right)-f_{n}\left(t_{1}, s_{1}, x_{1}, \cdots, x_{n}, y\right)\right|: t_{1}, s_{1}, t_{2}, s_{2} \in[0, T],\right. \\
& \left.\qquad\left|t_{2}-t_{1}\right| \leq \varepsilon,\left|s_{2}-s_{1}\right| \leq \varepsilon,\left|x_{i}\right| \leq r_{0},|y| \leq D_{1}\right\}, \\
& \left.\omega^{T}\left(x_{i}, \varepsilon\right)\right)=\sup \left\{\left|x_{i}\left(t_{2}, s_{2}\right)-x_{i}\left(t_{1}, s_{1}\right)\right|: t_{1}, s_{1}, t_{2}, s_{2} \in[0, T],\left|t_{2}-t_{1}\right| \leq \varepsilon,\left|s_{2}-s_{1}\right| \leq \varepsilon\right\}, \\
& \omega^{T}\left(k_{n}, \varepsilon\right)=\sup \left\{\left|k_{n}\left(t_{2}, s_{2}, v, w\right)-k_{n}\left(t_{1}, s_{1}, v, w\right)\right|:\right. \\
& \left.\qquad t_{1}, s_{1}, t_{2}, s_{2}, v, w \in[0, T],\left|t_{2}-t_{1}\right| \leq \varepsilon,\left|s_{2}-s_{1}\right| \leq \varepsilon\right\}, \\
& U_{r_{0}}^{T}=\sup \left\{\left|h_{n}\left(v, w,\left(x_{j}\right)_{j=1}^{\infty}\right)\right|: v, w \in[0, T], x_{i} \in\left[-r_{0}, r_{0}\right]\right\} .
\end{aligned}
$$

Since $x_{i}$ was an arbitrary element of $X_{i}$ for all $i \in \mathbb{N}$ in (4.7), we obtain

$$
\begin{aligned}
& \omega^{T}\left(H_{n}\left(\prod_{i=1}^{\infty} X_{i}\right)\right) \leq \varphi\left(\max _{1 \leq i \leq n} \omega^{T}\left(x_{i}, \varepsilon\right)\right)+\omega_{r_{0}, D_{1}}^{T}\left(f_{n}, \varepsilon\right)+T^{2} U_{r_{0}}^{T} \omega^{T}\left(k_{n}, \varepsilon\right) \\
& \quad+b_{n}\left(r_{0}\right) \int_{0}^{T} \int_{T}^{\infty}\left[\left|k_{n}\left(t_{2}, s_{2}, v, w\right)\right|+\left|k_{n}\left(t_{1}, s_{1}, v, w\right)\right|\right] a_{n}(v, w) d v d w \\
& \quad+b_{n}\left(r_{0}\right) \int_{T}^{\infty} \int_{0}^{\infty}\left[\left|k_{n}\left(t_{2}, s_{2}, v, w\right)\right|+\left|k_{n}\left(t_{1}, s_{1}, v, w\right)\right|\right] a_{n}(v, w) d v d w,
\end{aligned}
$$

and by the uniform continuity of $f_{n}$ and $k_{n}$ on the compact sets $[0, T] \times[0, T] \times$ $\left[-r_{0}, r_{0}\right]^{n} \times\left[-D_{1}, D_{1}\right]$ and $[0, T] \times[0, T] \times[0, T] \times[0, T]$ respectively, we have $\omega_{r_{0}, D_{1}}^{T}\left(f_{n}, \varepsilon\right) \longrightarrow 0$ and $\omega^{T}\left(k_{n}, \varepsilon\right) \longrightarrow 0$ as $\varepsilon \longrightarrow 0$. Therefore, we obtain

$$
\begin{gathered}
\omega^{T}\left(H_{n}\left(\prod_{i=1}^{\infty} X_{i}\right)\right) \leq \varphi\left(\max _{1 \leq i \leq n} \omega^{T}\left(x_{i}, \varepsilon\right)\right) \\
+b_{n}\left(r_{0}\right) \int_{0}^{T} \int_{T}^{\infty}\left[\left|k_{n}\left(t_{2}, s_{2}, v, w\right)\right|+\left|k_{n}\left(t_{1}, s_{1}, v, w\right)\right|\right] a_{n}(v, w) d v d w \\
+b_{n}\left(r_{0}\right) \int_{T}^{\infty} \int_{0}^{\infty}\left[\left|k_{n}\left(t_{2}, s_{2}, v, w\right)\right|+\left|k_{n}\left(t_{1}, s_{1}, v, w\right)\right|\right] a_{n}(v, w) d v d w .
\end{gathered}
$$

Now taking $T \longrightarrow \infty$ and by using assumption $\left(a_{6}\right)$ we get

$$
\omega_{0}\left(H_{n}\left(\prod_{i=1}^{\infty} X_{i}\right)\right) \leq \varphi\left(\max _{1 \leq i \leq n} \omega_{0}\left(X_{i}\right)\right) .
$$


On the other hand, for all $x_{i}, y_{i} \in X_{i}(i \in \mathbb{N})$ and $t, s \in \mathbb{R}_{+}$we get

$$
\begin{aligned}
& \left|H_{n}\left(\left(x_{i}\right)_{i=1}^{\infty}\right)(t, s)-H_{n}\left(\left(y_{i}\right)_{i=1}^{\infty}\right)(t, s)\right| \\
& \leq \varphi\left(\max _{1 \leq i \leq n}\left|x_{i}(t, s)-y_{i}(t, s)\right|\right) \\
& +\left|\int_{0}^{\infty} \int_{0}^{\infty} k_{n}(t, s, v, w)\left[h_{n}\left(v, w,\left(x_{i}(v, w)\right)_{i=1}^{\infty}\right)-h_{n}\left(v, w,\left(y_{i}(v, w)\right)_{i=1}^{\infty}\right)\right] d v d w\right| \\
& \leq \varphi\left(\max _{1 \leq i \leq n}\left|x_{i}(t, s)-y_{i}(t, s)\right|\right)+2 b_{n}\left(r_{0}\right) \int_{0}^{\infty} \int_{0}^{\infty} a_{n}(v, w)\left|k_{n}(t, s, v, w)\right| d v d w .
\end{aligned}
$$

Thus, we obtain

$$
\begin{aligned}
& \operatorname{diam}\left(H_{n}\left(\prod_{i=1}^{\infty} X_{i}\right)(t, s)\right) \\
& \quad \leq \varphi\left(\max _{1 \leq i \leq n} \operatorname{diam}\left(X_{i}(t, s)\right)\right)+2 b_{n}\left(r_{0}\right) \int_{0}^{\infty} \int_{0}^{\infty} a_{n}(v, w)\left|k_{n}(t, s, v, w)\right| d v d w .
\end{aligned}
$$

Taking $\|(t, s)\| \longrightarrow \infty$ in the inequality (4.9), then using $\left(a_{5}\right)$ we have

$$
\lim _{\|(t, s)\| \longrightarrow \infty} \operatorname{diam}\left(H_{n}\left(\prod_{i=1}^{\infty} X_{i}\right)(t, s)\right) \leq \varphi\left(\max _{1 \leq i \leq n} \lim _{(t, s) \| \longrightarrow \infty} \operatorname{diam}\left(X_{i}(t, s)\right)\right) .
$$

Further, combining (4.8) and (4.10) we get

$$
\begin{aligned}
\mu\left(H_{n}\left(\prod_{i=1}^{\infty} X_{i}\right)\right) & =\omega_{0}\left(H_{n}\left(\prod_{i=1}^{\infty} X_{i}\right)+\lim _{\|(t, s)\| \longrightarrow \infty} \operatorname{diam} H_{n}\left(\prod_{i=1}^{\infty} X_{i}\right)(t, s)\right. \\
& \leq \varphi\left(\max _{1 \leq i \leq n} \omega_{0}\left(X_{i}\right)\right)+\varphi\left(\max _{1 \leq i \leq n} \lim _{\| t, s) \| \longrightarrow \infty} \operatorname{diam}\left(X_{i}(t, s)\right)\right) \\
& \leq \varphi\left[\max _{1 \leq i \leq n}\left(\omega_{0}\left(X_{i}\right)+\lim _{\|(t, s)\| \longrightarrow \infty} \operatorname{diam}\left(X_{i}(t, s)\right)\right)\right] \\
& \leq 2 \varphi\left[\max _{1 \leq i \leq n} \frac{1}{2}\left(\omega_{0}\left(X_{i}\right)+\lim _{\|(t, s)\| \longrightarrow \infty} \operatorname{diam}\left(X_{i}(t, s)\right)\right)\right] .
\end{aligned}
$$

Let us consider the measure of noncompactness $\mu_{1}=\frac{1}{2} \mu$, thus we get

$$
\mu_{1}\left(H_{n}\left(\prod_{i=1}^{\infty} X_{i}\right)\right) \leq \varphi\left(\sup _{i} \mu_{1}\left(X_{i}\right)\right) .
$$

Now by using Theorem 8 , there exist $\left(x_{i}\right)_{i=1}^{\infty} \in\left(B C\left(\mathbb{R}_{+} \times \mathbb{R}_{+}\right)\right)^{\omega}$ such that

$$
=f_{n}\left(t, s, x_{1}(t, s) \ldots, x_{n}(t, s), \int_{0}^{\infty} \int_{0}^{\infty} k_{n}(t, s, v, w) h_{n}\left(v, w,\left(x_{i}(v, w)\right)_{i=1}^{\infty}\right) d v d w\right)
$$

and the proof is complete. 
Example 2. Consider the following system of functional integral equations

$$
\begin{gathered}
x_{n}(t, s)=\frac{t^{2 n} s^{2 n}}{t^{4 n} s^{4 n}+1}+\tanh \left(\frac{1}{n} \sum_{i=1}^{n}\left|x_{i}(t, s)\right|\right) \\
+\int_{0}^{\infty} \int_{0}^{\infty} \frac{e^{-n w}\left(e^{-n v}-1\right)}{2^{n}\left[t^{n} s^{n}+1\right]} \sum_{i=1}^{\infty} e^{-i v \sqrt[2 n-1]{\tanh x_{i}(v, w)}} d v d w
\end{gathered}
$$

Eq. (4.11) is a special case of Eq. (1.2) where

$$
\begin{aligned}
& f_{n}\left(t, s, x_{1}, \ldots, x_{n}, z\right)=\frac{t^{2 n} s^{2 n}}{t^{4 n} s^{4 n}+1}+\tanh \left(\frac{1}{n} \sum_{i=1}^{n}\left|x_{i}\right|\right)+z, \\
& k_{n}(t, s, v, w)=\frac{e^{-n w}\left(e^{-n v}-1\right)}{2^{n}\left[t^{n} s^{n}+1\right]} \\
& h_{n}\left(t, s,\left(x_{j}\right)_{j=1}^{\infty}\right)=\sum_{i=1}^{\infty} e^{-i t \sqrt[2 n-1]{\tanh x_{i}}}, \\
& a_{n}(t, s)=\frac{1}{e^{t}-1}, b_{n}(r)=\sqrt[2 n-1]{\tanh r} .
\end{aligned}
$$

Suppose that $t, s \in \mathbb{R}_{+}$. Now, by taking $\varphi(t)=\tanh (t)$ we have

$$
\begin{aligned}
& \left|f_{n}\left(t, s, x_{1}, \ldots, x_{n}, u\right)-f_{n}\left(t, s, y_{1}, \ldots, y_{n}, v\right)\right| \\
& \quad \leq\left|\tanh \left(\frac{1}{n} \sum_{i=1}^{n}\left|x_{i}\right|\right)-\tanh \left(\frac{1}{n} \sum_{i=1}^{n}\left|y_{i}\right|\right)\right|+|u-v| \\
& \quad \leq \tanh \left(\frac{1}{n} \sum_{i=1}^{n}\left|x_{i}-y_{i}\right|\right)+|u-v| \\
& \quad \leq \tanh \left(\max _{1 \leq i \leq n}\left|x_{i}-y_{i}\right|\right)+|u-v| \\
& \left.\quad=\varphi\left(\max _{1 \leq i \leq n}\left|x_{i}-y_{i}\right|\right)+|u-v|\right) .
\end{aligned}
$$

Thus, we infer that condition $\left(a_{1}\right)$ holds. On the other hand

$$
\begin{aligned}
M & =\sup \left\{\left|f_{n}(t, s, 0, \ldots, 0)\right|: t, s \in \mathbb{R}_{+}, n \in \mathbb{N}\right\} \\
& =\sup \left\{\frac{t^{2 n} s^{2 n}}{t^{4 n} s^{4 n}+1}: t, s \in \mathbb{R}_{+}, n \in \mathbb{N}\right\}=\frac{1}{2}
\end{aligned}
$$

which shows the condition $\left(a_{2}\right)$ is valid. Also, assumption $\left(a_{3}\right)$ clearly is evident. In order to show that assumption $\left(a_{4}\right)$ is satisfied, let us assume that $\sup _{1 \leq i<\infty}\left|x_{i}\right|<\infty$, so we have

$$
\left|h_{n}\left(t, s,\left(x_{j}\right)_{j=1}^{\infty}\right)\right|=\sum_{i=1}^{\infty} e^{-i t \sqrt[2 n-1]{\tanh x_{i}}}
$$




$$
\begin{aligned}
& \leq \frac{1}{e^{t}-1} \sqrt[2 n-1]{\tanh \left(\sup _{1 \leq i<\infty}\left|x_{i}\right|\right)} \\
& =a_{n}(t, s) b_{n}\left(\sup _{1 \leq i<\infty}\left|x_{i}\right|\right) .
\end{aligned}
$$

On the other hand, the function $(v, w) \longrightarrow a_{n}(v, w) k_{n}(v, w, t, s)$ is integrable over $\mathbb{R}_{+} \times \mathbb{R}_{+}$for any fixed $t, s \in \mathbb{R}_{+}$and $n \in \mathbb{N}$. Thus, condition $\left(a_{4}\right)$ is valid. Further, we get:

$$
\begin{aligned}
D & =\sup \left\{\frac{1}{2^{n}\left[t^{n} s^{n}+1\right]} \int_{0}^{\infty} \int_{0}^{\infty}\left|\frac{e^{-n w}\left(e^{-n v}-1\right)}{e^{v}-1}\right| d v d w: t, s \in \mathbb{R}_{+}, n \in \mathbb{N}\right\} \\
& =\sup \left\{\frac{1}{2^{n}\left[t^{n} s^{n}+1\right]}\right. \\
& \left.\quad \int_{0}^{\infty} \int_{0}^{\infty}\left|e^{-n w}\left(e^{-v}+e^{-2 v}+\ldots+e^{-n v}\right)\right| d v d w: t, s \in \mathbb{R}_{+}, n \in \mathbb{N}\right\} \\
= & \sup \left\{\frac{1}{2^{n}\left[t^{n} s^{n}+1\right] n}\left(1+\frac{1}{2}+\frac{1}{3}+\ldots+\frac{1}{n}\right): t, s \in \mathbb{R}_{+}, n \in \mathbb{N}\right\} \\
= & \frac{1}{2}<\infty
\end{aligned}
$$

and

$$
\lim _{\|t, s\| \rightarrow \infty} \frac{1}{2^{n}\left[t^{n} s^{n}+1\right]} \int_{0}^{\infty} \int_{0}^{\infty} \frac{e^{-n w}\left(e^{-n v}-1\right)}{e^{v}-1} d v d w=0 .
$$

This implies that the condition $\left(a_{5}\right)$ holds. Moreover, for arbitrarily fixed $T>0$ we obtain:

$$
\int_{T}^{\infty} \int_{0}^{T} a_{n}(v, w)\left|k_{n}(t, s, v, w)\right| d v d w \leq \frac{e^{-n T}}{2^{n} n}\left(n-e^{-T}-\frac{e^{-2 T}}{2}-\ldots-\frac{e^{-n T}}{n}\right)
$$

and

$$
\int_{0}^{\infty} \int_{T}^{\infty} a_{n}(v, w)\left|k_{n}(t, s, v, w)\right| d v d w \leq \frac{1}{2^{n} n}\left(e^{-T}+\frac{e^{-2 T}}{2}+\ldots+\frac{e^{-n T}}{n}\right) .
$$

From the above estimate, we infer that condition $\left(a_{6}\right)$ is valid. It is easy to see that each number $r \geq 4$ satisfies the inequality in condition $\left(a_{7}\right)$, i.e.,

$$
\varphi(r)+M+D b_{n}(r)=\tanh (r)+\frac{1}{2}+\frac{1}{2} \sqrt[2 n-1]{r} \leq r .
$$

Thus, as the number $r_{0}$ we can take $r_{0}=4$. Consequently, all the conditions of Theorem 9 are satisfied. Hence the system of functional integral equations (4.11) has at least one solution which belong to the space $\left(B C\left(\mathbb{R}_{+} \times \mathbb{R}_{+}\right)\right)^{\omega}$. 


\section{REFERENCES}

[1] R. P. Agarwal, M. Meehan, and D. O'Regan, Fixed point theory and applications. Cambridge university press, 2001, vol. 141.

[2] A. Aghajani, M. Mursaleen, and A. S. Haghighi, "Fixed point theorems for Meir-Keeler condensing operators via measure of noncompactness," Acta Mathematica Scientia, vol. 35, no. 3, pp. 552-566, 2015.

[3] A. Aghajani, R. Allahyari, and M. Mursaleen, "A generalization of Darbo's theorem with application to the solvability of systems of integral equations," Journal of Computational and Applied Mathematics, vol. 260, pp. 68-77, 2014.

[4] A. Aghajani, J. Banaś, and Y. Jalilian, "Existence of solutions for a class of nonlinear volterra singular integral equations," Computers \& Mathematics with Applications, vol. 62, no. 3, pp. 1215-1227, 2011.

[5] R. Arab, R. Allahyari, and A. S. Haghighi, "Existence of solutions of infinite systems of integral equations in two variables via measure of noncompactness," Applied Mathematics and Computation, vol. 246, pp. 283-291, 2014.

[6] S. Banach, "Sur les opérations dans les ensembles abstraits et leur application aux équations intégrales," Fund. Math, vol. 3, no. 1, pp. 133-181, 1922.

[7] J. Banaś, K. Balachandran, and J. Diana, "Existence and global attractivity of solutions of a nonlinear functional integral equation," Applied Mathematics and Computation, vol. 216, no. 1, pp. 261-268, 2010.

[8] J. Banaś and K. Goebel, "Measures of noncompactness in banach spaces," Lecture Notes in Pure and Applied Mathematics, Marcel Dekker, Inc, vol. 60, 1980.

[9] J. Banaś and M. Mursaleen, Sequence Spaces and Measures of Noncompactness with Applications to Differential and Integral Equations. Springer India, 2014, http://dx.doi.org/10.1007/978-81322-1886-9.

[10] J. Banaś, D. O’Regan, and K. Sadarangani, "On solutions of a quadratic hammerstein integral equation on an unbounded interval," Dynamic Systems and Applications, vol. 18, no. 2, p. 251, 2009.

[11] J. Banaś and B. Rzepka, "On solutions of infinite systems of integral equations of Hammerstein type," J. Nonlin. Convex Anal., vol. 18, pp. 261-278, 2017.

[12] M. Cichoń and M. M. Metwali, "On monotonic integrable solutions for quadratic functional integral equations," Mediterranean Journal of Mathematics, pp. 1-18, 2013.

[13] G. "Darbo's", "Punti uniti in trasformazioni a codominio non compatto," Rendiconti del Seminario Matematico della Universita di Padova, vol. 24, pp. 84-92, 1955.

[14] M. A. Darwish, "On quadratic integral equation of fractional orders," Journal of Mathematical Analysis and Applications, vol. 311, no. 1, pp. 112-119, 2005.

[15] B. Dhage, "Nonlinear quadratic first order functional integro-differential equations with periodic boundary conditions," Dynamic Systems and Applications, vol. 18, no. 2, p. 303, 2009.

[16] B. Dhage and M. Imdad, "Asymptotic behaviour of nonlinear quadratic functional integral equations involving carathéodory," Nonlinear Analysis: Theory, Methods \& Applications, vol. 71, no. 12, pp. e1285-e1291, 2009.

[17] J. Dugundji, "Topology," Ally and Bacon, Boston, 1966.

[18] J. Garcia-Falset, "Existence of fixed points and measures of weak noncompactness," Nonlinear Analysis: Theory, Methods \& Applications, vol. 71, no. 7, pp. 2625-2633, 2009.

[19] J. Banaś and J. Rocha Martin and Sadarangani, Kishin B, "On solutions of a quadratic integral equation of hammerstein type," Mathematical and Computer Modelling, vol. 43, no. 1-2, pp. 97 104, 2006. 
[20] T.-C. Lim, "On characterizations of Meir-Keeler contractive maps," Nonlinear Analysis: Theory, Methods \& Applications, vol. 46, no. 1, pp. 113-120, 2001.

[21] A. Meir and E. Keeler, "A theorem on contraction mappings," Journal of Mathematical Analysis and Applications, vol. 28, no. 2, pp. 326-329, 1969.

[22] M. Mursaleen and S. Mohiuddine, "Applications of measures of noncompactness to the infinite system of differential equations in $\ell \mathrm{p}$ spaces," Nonlinear Analysis: Theory, Methods \& Applications, vol. 75, no. 4, pp. 2111-2115, 2012.

[23] M. Mursaleen, "Application of measure of noncompactness to infinite system of differential equations," Canadian Math. Bull, 2011.

[24] L. Olszowy, "Solvability of some functional integral equation," Dynamic Systems and Applications, vol. 18, no. 3, p. 667, 2009.

[25] L. Olszowy, "Solvability of infinite systems of singular integral equations in Fréchet space of continuous functions," Computers \& Mathematics with Applications, vol. 59, no. 8, pp. 2794 2801, 2010.

[26] T. Suzuki, "Fixed-point theorem for asymptotic contractions of Meir-Keeler type in complete metric spaces," Nonlinear Analysis: Theory, Methods \& Applications, vol. 64, no. 5, pp. 971-978, 2006.

Authors' addresses

Mohsen Hosseinzadeh Moghaddam

Department of Mathematics, Mashhad Branch, Islamic Azad University, Mashhad, Iran.

E-mail address: mohsen11124@gmail.com

Reza Allahyari

Department of Mathematics, Mashhad Branch, Islamic Azad University, Mashhad, Iran. (Corresponding author)

E-mail address: rezaallahyariemshdiau.ac.ir

Mohsen Erfanian Omidvar

Department of Mathematics, Mashhad Branch, Islamic Azad University, Mashhad, Iran.

E-mail address: erfanian@mshdiau.ac.ir

Mahmoud Hassani

Department of Mathematics, Mashhad Branch, Islamic Azad University, Mashhad, Iran.

E-mail address: hassani@mshdiau.ac.ir 